\title{
COBORDISM MASSEY PRODUCTS
}

\author{
BY \\ J. C. ALEXANDER
}

\begin{abstract}
The structure of Massey products is introduced into the bordism ring $\Omega^{s}$ of manifolds with structure $S$ and machinery is developed to investigate it. The product is changed to one in homotopy via the Pontrjagin-Thom map and methods for computation via the Adams spectral sequence are developed. To illustrate the methods, some products in $\Omega^{s U}$ and $\Omega^{S p}$ are computed.
\end{abstract}

1. Introduction. In [18] and [8], W. Massey introduced secondary products into the cohomology ring of a space. The construction works for any associative differential ring and such products exist in many contexts. Secondary products have also been introduced by $\mathrm{H}$. Toda in stable homotopy theory [17] and in a more general context by E. Spanier [15]. In the situation of cobordism of manifolds, we almost have a differential ring. Products do not exist (they have corners) and there is no notion of subtraction. However, as might be guessed from the existence of this paper, the obstructions to defining a meaningful secondary product are not insurmountable. In this paper, we develop such products.

The idea of secondary cobordism products has been in the air for some time. They have been mentioned by various workers in the field, but not really used as such. Also, G. Porter has developed secondary products in generalized cohomologies [12]; cobordism is of course a special case. The point of view in this paper is somewhat different than Porter's in that we consider Massey products as a way of constructing, in some sense, manifolds to represent cobordism classes.

We are particularly interested in ordinary triple products and limit ourselves to discussing them. The construction immediately generalizes to matric products [9], and presumably at least some of it generalizes to higher products [6]. The cost of doing so explicitly is, at the least, more complex notation. We leave it to the reader to pursue these if he wishes. Matric products enter unbidden in $\$ 5$; we briefly discuss them there.

In $\S 2$, we isolate the algebraic structure we want to study. Here we set up the notation and properties of secondary products. In $\$ 3$ we define cobordism Massey products. In place of subtraction we have glueing, and we must carefully develop the notion of structured manifold so we may glue them together. We also define

Received by the editors June 17, 1970 and, in revised form, May 17, 1971.

AMS 1970 subject classifications. Primary 57D90, 57D75, 55G30; Secondary 55H15, 55E10.

Key words and phrases. Cobordism, Massey product, manifolds, Pontrjagin-Thom map, Adams spectral sequence. 
products and some other auxiliary constructions. Thus we do not get to the basic definition until 3.7.

The idea is simple. If we have closed manifolds $L, M, N$ such that $L M=\partial A$, $M N=\partial B$, we glue $A N$ and $L B$ along their common boundary $L M N$ to get $X$. The classes of such $X$ are the Massey product.

In $\$ 4$ we change the problem into one of homotopy. We define a secondary structure on the homotopy of the Thom spectrum [Definition 4.1] and show that the Pontrjagin-Thom map is a map of secondary structures [Theorem 4.3]. Again the definition is simple. If we have maps $f, g, h$ representing homotopy elements such that $f \wedge g, g \wedge h$ represent zero, we put cones $F$ on $f \wedge g$ and $G$ on $g \wedge h$ and glue $F \wedge h$ and $f \wedge G$ together along $f \wedge g \wedge h$. The resulting map $H$ determines a homotopy element and the set of such $H$ determines the secondary product. We remark that matric and higher products could be defined here and they would have the obvious properties.

In $\$ 5$ we develop our main computational tool-the Adams spectral sequence. The results we get are analogous to those of May [9] and Moss [10].

The secondary structure on unoriented and complex cobordism is nonexistent since these rings are polynomial. In oriented cobordism, most, if not all, of the secondary products are trivial. In $\$ 6$, we compute some of the secondary structures for $S U$ and $S p$ cobordism.

2. Secondary products. In this section we make explicit the algebraic structure we want to consider. Let $R=\left\{R_{n}\right\}$ be a graded associative ring with unit. Let $k= \pm 1$. Let $a, b, c, d, e$ be homogeneous elements of $R$ of gradings $\alpha, \beta, \gamma, \delta, \varepsilon$ respectively.

2.1. Definition. $R$ has a secondary (or Massey) product structure (of degree $k$ ) if there is assigned to any $a, b, c$ satisfying $a b=0, b c=0$, a set of elements, denoted $\langle a, b, c\rangle, \subset R_{\alpha+\beta+\gamma+k}$. This set of elements is called the secondary product of $a, b, c$ (in that order). It is to be a coset of the group $a \cdot R_{\beta+\gamma+k}+R_{\alpha+\beta+k} \cdot c$, which is called the indeterminacy of $\langle a, b, c\rangle$, denoted indet $\langle a, b, c\rangle$. The structure is to be governed by the following axioms.

1. If any of $a, b, c$ is zero, $\langle a, b, c\rangle=$ indet $\langle a, b, c\rangle$.

2. $\langle a, b, c\rangle$ is linear. That is, if $\langle a, b, c\rangle,\langle d, b, c\rangle$ are defined and $\alpha=\delta$, then $\langle a+d, b, c\rangle$ is defined and $\langle a+d, b, c\rangle \subset\langle a, b, c\rangle+\langle d, b, c\rangle$. Similar equations hold for the second and third entries.

3. If $\langle a, b, c\rangle$ is defined, $d\langle a, b, c\rangle \subset(-1)^{\delta}\langle d a, b, c\rangle,\langle a, b, c\rangle d \subset\langle a, b, c d\rangle$.

4. If $\langle a d, b, c\rangle$ is defined, $\langle a d, b, c\rangle \subset\langle a, d b, c\rangle$. If $\langle a, b, d c\rangle$ is defined, $\langle a, b, d c\rangle$ $\subset\langle a, b d, c\rangle$.

5. If all terms below are defined, $0 \in\langle a, b, c\rangle d+(-1)^{\alpha} a\langle b, c, d\rangle$.

6. If there exists a choice of $y \in\langle b, c, d\rangle$ so that $\langle a, y, e\rangle$ is defined, there exist (by 2.1.5) choices $x \in\langle a, b, c\rangle, z \in\langle c, d, e\rangle$ so that all terms below are defined and $0 \in(-1)^{\alpha}\langle x, d, e\rangle+\langle a, y, e\rangle+(-1)^{\beta}\langle a, b, z\rangle$. 
The structure is said to be commutative if $R$ is commutative as a ring and

7. If $\langle a, b, c\rangle$ is defined, $\langle a, b, c\rangle=(-1)^{\alpha \beta+\beta \gamma+\gamma \alpha}\langle c, b, a\rangle$.

8. If $a b=b c=c a=0,0 \in(-1)^{\alpha \gamma}\langle a, b, c\rangle+(-1)^{\beta \alpha}\langle b, c, a\rangle+(-1)^{\gamma \beta}\langle c, a, b\rangle$.

$A$ ring map $f: R \rightarrow R^{\prime}$ of degree 0 is a map of secondary structures if, whenever $\langle a, b, c\rangle$ is defined, $f\langle a, b, c\rangle \subset\langle f a, f b, f c\rangle$.

If $R$ is bigraded, we assume that $a, b, c, d, e$ are bihomogeneous. $k$ becomes $\left(k^{\prime}, k^{\prime \prime}\right)$ with $k^{\prime}+k^{\prime \prime}= \pm 1 . \alpha, \beta, \gamma, \delta, \varepsilon$ are total degrees. The product $\langle a, b, c\rangle$ is contained in a bihomogeneous component.

We thus have categories of rings with secondary structures. An isomorphism is a map which is an isomorphism on the underlying sets. Note, however, that these categories do not have sub or quotient objects.

It is clear that we could have an external secondary product. Thus suppose we have six graded abelian groups $R_{1}, \ldots, R_{8}$ and maps $\mu_{12}: R_{1} \otimes R_{2} \rightarrow R_{4}, \mu_{23}$ : $R_{2} \otimes R_{3} \rightarrow R_{5}, \mu_{15}: R_{1} \otimes R_{5} \rightarrow R_{6}, \mu_{43}: R_{4} \otimes R_{3} \rightarrow R_{6}$ satisfying

$$
\mu_{43}\left(\mu_{12} \otimes 1\right)=\mu_{15}\left(1 \otimes \mu_{23}\right): R_{1} \otimes R_{2} \otimes R_{3} \rightarrow R_{6} .
$$

For elements $a \in R_{1}, b \in R_{2}, c \in R_{3}$ satisfying $\mu_{12}(a \otimes b)=0, \mu_{23}(b \otimes c)=0$, there will be assigned a set of elements $\langle a, b, c\rangle \subset R_{6}$. These will satisfy the analogues of 2.1.1 through 2.1.6. We will pursue the geometric analogue of this situation in $\S 5$.

We finish this section by listing the standard examples.

2.2. EXAMPLES. 1. The trivial secondary structure. For any $a, b, c$ with $a b=0$, $b c=0$, let $\langle a, b, c\rangle=$ indet $\langle a, b, c\rangle$.

2. The negative of a structure. If $\langle a, b, c\rangle \subset R_{n}$, let $\langle a, b, c\rangle^{\prime}=(-1)^{n}\langle a, b, c\rangle$. Then $\langle,,\rangle^{\prime}$ is a secondary product, called the negative of $\langle,$,$\rangle .$

3. Massey product. See [18]. This works for any associative differential ring.

4. Toda bracket. See [17] and more generally [10].

In the next section, we put such a structure on cobordism rings.

3. Cobordism Massey products. Before we can make our definition, we must collect some machinery. We recall the notion of structured manifolds, how to form their products and how to glue them together. All of this is fairly standard. For example, see [16].

Let $B O(n)$ be a classifying space (say a Grassmannian) for $O(n)$, the orthogonal group on $n$-dimensional Euclidean space $E^{n}$. Let $f_{n}: B(n) \rightarrow B O(n)$ be a fibration. We suppose we have a sequence of inclusions $i_{n}: B(n) \rightarrow B(n+1), j_{n}: B O(n)$ $\rightarrow B O(n+1)$ such that $f_{n+1} i_{n}=j_{n} f_{n}$. Let $H^{+}$(resp. $\left.H^{-}\right)=\left\{t \in E^{1} \mid t \geqq 0\right.$ (resp. $\left.\left.\leqq 0\right)\right\}$ denote a half-line.

3.1. Definition. $A$ B-structured manifold is a class of triples $(M, \iota, \xi)$ where $M$ is a compact smooth manifold of dimension $m, \iota: M \rightarrow H^{+} \times E^{m+k-1}$ is an embedding such that

(1) $\iota \mid \partial M: \partial M \rightarrow E^{m+k-1}=0 \times E^{m+k-1}$, 
(2) there exists a tubular neighborhood of $\partial M, \partial M \times[0, \varepsilon) \subset M$ such that $\iota(x, t)$ $=(t, \iota x)$ for $t \in[0, \varepsilon) \subset H^{+}, x \in \partial M$,

and $\xi: M \rightarrow B(k)$ is a map such that $f_{n} \xi=\nu$, the normal bundle of $\iota$. We identify various $(M, \iota, \xi)$ gotten by varying $k$ and using $i_{k}, j_{k}$.

The boundary of $(M, \iota, \xi)$ is $(\partial M, \iota|\partial M, \xi| \partial M)$.

We often drop some of the notation and refer to the $B$ manifold $M$. For $M$ closed, we let $-M$ denote $M$ with the "opposite" structure. $M$ and $M^{\prime}$ are cobordant if $M \cup-M^{\prime}=\partial N$ for some $N$.

In the usual way, we form the cobordism groups $\Omega_{m}^{B}$ of $B$-manifolds. $[M] \in \Omega_{m}^{B}$ denotes the class of $M .-[M]=[-M]$.

3.2. Definition. If $\boldsymbol{B}^{\prime}=\left\{\boldsymbol{B}^{\prime}(n), i_{n}^{\prime}, f_{n}^{\prime}\right\}$ is another structure, a map $F: B \rightarrow B^{\prime}$ is a sequence of maps $F_{n}: B(n) \rightarrow B^{\prime}(n)$ such that $f_{n}^{\prime} F_{n}=f_{n}, i_{n}^{\prime} F_{n}=F_{n} i_{n}$. Such an $F$ induces a homomorphism $F_{*}: \Omega_{m}^{B} \rightarrow \Omega_{m}^{B^{\prime}}$.

There exist standard maps $\mu_{B O}: B O(k) \times B O\left(k^{\prime}\right) \rightarrow B O\left(k+k^{\prime}\right)$ such that $\mu_{B O}\left(j_{k} \times 1\right)=\mu_{B O}\left(1 \times j_{k^{\prime}}\right)=j_{k+k^{\prime}} \mu_{B^{\prime}}$.

3.3. Definition. $B$ is multiplicative if there exists $\mu_{B}: B(k) \times B\left(k^{\prime}\right) \rightarrow B\left(k+k^{\prime}\right)$ such that $\mu_{B O}\left(f_{k} \times f_{k^{\prime}}\right)=f_{k+k^{\prime}} \mu_{B}, \mu_{B}\left(i_{k} \times 1\right)=\mu_{B}\left(1 \times i_{k^{\prime}}\right)=i_{k+k^{\prime}} \mu_{B} . B$ is associative (commutative) if $\mu_{B}$ is associative (commutative).

If $B$ is multiplicative, $\Omega^{B}=\left\{\Omega_{m}^{B}\right\}$ has the structure of a graded ring; it is associative or commutative if $B$ is. Henceforth we assume that $B$ is associatively multiplicative. (We will also proceed to modify this assumption in 3.9.) We suppose all maps $F$ are compatible with the multiplication.

We can now turn to the question of products. Suppose $M=(M, \iota, \xi)$, $M^{\prime}=\left(M^{\prime}, \iota^{\prime}, \xi^{\prime}\right)$ are $B$-manifolds, both possibly with boundary. Then

$$
\begin{gathered}
\iota: M \rightarrow H^{+} \times E^{m+k-1}, \quad \iota^{\prime}: M^{\prime} \rightarrow H^{+} \times E^{m^{\prime}+k^{\prime}-1}, \\
\iota \times \iota^{\prime}: M \times M^{\prime} \rightarrow H^{+} \times E^{m+k-1} \times H^{+} \times E^{m^{\prime}+k^{\prime}-1}=H^{+} \times H^{+} \times E^{m+m^{\prime}+k+k^{\prime}-2} .
\end{gathered}
$$

In polar coordinates $H^{+} \times H^{+}=\{(r, \theta) \mid 0 \leqq \theta \leqq \pi / 2\}$. We define $\lambda: H^{+} \times H^{+}$ $\rightarrow H^{+} \times E^{1}$ by $\lambda(r, \theta)=(r, 2 \theta) . \lambda$ is a homeomorphism and a diffeomorphism except at the origin. $(\lambda \times 1)\left(\iota \times \iota^{\prime}\right): M \times M^{\prime} \rightarrow H^{+} \times E^{m+m^{\prime}+k+k^{\prime}-1}$ is an embedding; let $\tilde{M}$ be $M \times M^{\prime}$ with the induced differentiable structure.

3.4. Definition. The product $M M^{\prime}$ is the triple $\left(\tilde{M},(\lambda \times 1)\left(\iota \times \iota^{\prime}\right), \mu_{B}\left(\xi \times \xi^{\prime}\right)\right)$. $M M^{\prime}$ is a $B$-manifold.

We now consider glueing. We make two definitions. The first is the straightforward definition we will use in constructing secondary products; the second is a more intricate definition we will need in auxiliary constructions. (It is used to "undo" the effects of 3.4.)

Suppose $M=(M, \imath, \xi), M^{\prime}=\left(M^{\prime}, \iota^{\prime}, \xi^{\prime}\right)$ are two $B$-manifolds such that $\partial M=\partial M^{\prime}$. By this we mean there exists a diffeomorphism $\eta: \partial M \rightarrow \partial M^{\prime}$ such that $\iota^{\prime}=\imath \eta$, $\xi^{\prime}=\xi \eta$. Let $\kappa: H^{+} \rightarrow H^{-}$be the map $\kappa(t)=-t$.

3.5. Definition. $M \cup_{\eta} M^{\prime}\left(\right.$ or $\left.M \cup_{\partial M} M^{\prime}\right)$ is the triple $(\tilde{M}, \tilde{\imath}, \tilde{\xi})$ where 
$\tilde{M}=M \cup M^{\prime}$ with the identifications $\eta x \equiv x$ for $x \in \partial M, \tilde{\imath}=\iota \cup \kappa \iota^{\prime}, \quad \xi=\xi \cup \xi^{\prime}$. $\tilde{M}$ has the differentiable structure induced by $\tilde{\imath} . M \cup_{\eta} M^{\prime}$ is a closed B-manifold. It is $M, M^{\prime}$ glued along $\partial M=\partial M^{\prime}$.

Note that if $M, M^{\prime}$ are as in $3.4, \partial\left(M M^{\prime}\right)=\partial M \cdot M^{\prime} \cup_{\partial M \cdot \partial M^{\prime}} M \cdot \partial M^{\prime}$.

To define the more complicated glueing we need a map. Let

$$
H^{+} \times E^{1}=\{(r, \theta) \mid 0 \leqq \theta \leqq \pi\}, \quad H^{-} \times E^{1}=\{(r, \theta) \mid \pi \leqq \theta \leqq 2 \pi\} .
$$

Let

$$
\left(H^{+} \times E^{1}\right) \cup^{\prime}\left(H^{-} \times E^{1}\right)
$$

be the disjoint union. Let

$$
\lambda^{\prime}:\left(H^{+} \times E^{1}\right) \cup^{\prime}\left(H^{-} \times E^{1}\right) \rightarrow H^{+} \times E^{1}
$$

be given by $\lambda^{\prime}(r, \theta)=(r, \theta / 2)$.

3.6. Definition. Suppose we are given the following data:

(1) B-manifolds $(M, \iota, \xi),\left(M^{\prime}, \iota^{\prime}, \xi^{\prime}\right)$ of dimension $m$.

(2) A smooth manifold $L$ of dimension $m-1$ (possibly with boundary) and diffeomorphisms $g: L \rightarrow N \subset \partial M, g^{\prime}: L \rightarrow N^{\prime} \subset \partial M^{\prime}$, such that

(a) $\imath g=\imath^{\prime} g^{\prime}, \xi g=\xi^{\prime} g^{\prime}$;

(b) if $\partial L \neq \varnothing$, there exist tubular neighborhoods $(g \partial L) \times(-\varepsilon, \varepsilon) \subset \partial M$ and $\left(g^{\prime} \partial L\right)$ $\times(-\varepsilon, \varepsilon) \subset \partial M^{\prime}$ such that, for $x \in g \partial L, x^{\prime} \in g^{\prime} \partial L, t \in(-\varepsilon, \varepsilon) \subset E^{1}$,

(i) $(x, t) \in N$ if $t \leqq 0 ;\left(x^{\prime}, t\right) \in N^{\prime}$ if $t \leqq 0$.

(ii) $\iota(x, t)=(t, \iota x) \subset E^{1} \times E^{m+k-1}=0 \times E^{1} \times E^{m+k-1} \subset H^{+} \times E^{1} \times E^{m+k-1}$.

Let $\tilde{M}=M \cup M^{\prime}$ with identification $g x \equiv g^{\prime} x^{\prime}$ for $x \in L$. $\tilde{\imath}=\left(\lambda^{\prime} \times 1\right)\left(\iota \cup \kappa \iota^{\prime}\right)$. $\xi=\xi \cup \xi^{\prime} .(\tilde{M}, \tilde{\imath}, \xi)$ is a B-manifold denoted $M \cup_{L} M^{\prime}$.

If $N=\partial M, N^{\prime}=\partial M^{\prime}, 3.6$ reduces to 3.5 .

$$
\partial\left(M \cup_{L} M^{\prime}\right)=(\partial M-N) \cup_{\partial L}\left(\partial M^{\prime}-N^{\prime}\right) .
$$

We are now ready to make our main construction. Let $L, M, N$ be three $B$ manifolds such that $L M=\partial A, M N=\partial C$. Let $X=A N \cup_{L M N} L B$ (according to 3.5). $X$ is what we want to call the secondary product; we must check how well defined it is.

Suppose $L M=\partial A^{\prime}$ and $X^{\prime}=A^{\prime} N \cup_{L M N} L B . \tilde{A}=A^{\prime} \cup_{L M} A$ is closed and $X^{\prime} \cup-X$ $=\tilde{A} N$. Thus $\left[X^{\prime}\right]=[X]+[\tilde{A} N]$. Conversely, if $\tilde{A}$ is closed and $A^{\prime}=A \cup \tilde{A}, L M$ $=\partial A^{\prime}$. If $X^{\prime}=A^{\prime} N \cup_{L M N} L C,\left[X^{\prime}\right]=[X]+[\tilde{A} N]$. Changing $B$ causes a similar effect. Thus we know the indeterminacy.

Now suppose we change $L$ by a cobordism. Let $L^{\prime} \cup-L=\partial D$. Then if $A^{\prime}$ $=A \cup_{L M} D M, \partial A^{\prime}=L^{\prime} M$. Let $X^{\prime}=A^{\prime} N \cup_{L^{\prime} M N} L^{\prime} B$. Let $Y=D B \cup(X \times I)$ with glueing (by 3.6) along $A N \subset \partial D B$ and $A N \subset X \times 0 \subset \partial(X \times I)$. Here $I=[0,1]$ and the structure maps $(\iota, \xi)$ on $X \times I$ are the projections onto $X$ followed by the structure maps of $X$. If the codimension is large enough, we can approximate the map $Y \rightarrow E^{k}$ by an embedding and, if $X$ is identified with $X \times 1, \partial Y=X^{\prime} \cup-X$. Thus $X^{\prime}, X$ are cobordant. A cobordism of $N$ is handled similarly. 
Suppose $M^{\prime} \cup-M=\partial D$. Let $A^{\prime}=A \cup_{L M} L D, B^{\prime}=B \cup_{L M} D N$. Then $\partial A^{\prime}=L M^{\prime}$, $\partial B^{\prime}=M^{\prime} N$. Hence $X^{\prime}=A^{\prime} N \cup_{L M^{\prime} N} L B^{\prime}$. Let $Y=(A N \times I) \cup(L B \times I) \cup(L D N \times I)_{1}$ $\cup(L D N \times I)_{2} \cup(X \times I)$, with the following glueings:

(1) $L M N \times I \subset \partial(A N \times I)$ with $L M N \times I \subset \partial(L D N \times I)_{1}$,

(2) $L M N \times I \subset \partial(L D N \times I)_{2}$ with $L M N \times I \subset \partial(L B \times I)$,

(3) $L D N \times 1 \subset \partial(L D N \times I)_{1}$ with $L D N \times 1 \subset \partial(L D N \times I)_{2}$,

(4) $L M^{\prime} N \times I \subset \partial(L D N \times I)_{1}$ with $L M^{\prime} N \times I \subset \partial(L D N \times I)_{2}$,

(5) $X=X \times 0 \subset \partial(X \times I)$ with

$$
X=A N \cup_{L M N} L B=(A N \times 1) \cup_{L M N \times 1}(L B \times 1) \subset \partial(A N \times I) \cup \partial(L B \times I) .
$$

Then if the map $Y \rightarrow E^{k}$ is approximated by an embedding, $\partial Y=X^{\prime} \cup-X$ where $X^{\prime}=(A N \times 0) \cup(L B \times 0) \cup(L D N \times 0)_{1} \cup(L D N \times 0)_{2}$ with appropriate glueings and $X=X \times 1$.

Thus we have shown that the following is well defined and has the proper indeterminacy.

3.7. Definition. Let the set of $[X]=\langle[L],[M],[N]\rangle$.

3.8. THEOREM. 3.7 defines a secondary product structure of degree +1 on $\Omega^{B}$. If $B$ is commutative, so is the secondary structure. A multiplicative map induces a map of rings with secondary products.

To prove the theorem it is necessary to establish 2.1.1 through 2.1.8. These proofs consist of more glueings similar to those preceding the definition. We leave them to the reader.

3.9. Remarks ON Generalizations. The construction given here works equally well for other types of manifolds. In fact, it works even better in the piecewise-linear or topological category, since the problems with corners do not enter. We could also, with a small amount of extra work, consider manifolds with groups acting on them [4].

Furthermore, in some of the more interesting cases, such as symplectic cobordism, the structure $B$ is not associative, but only homotopy associative. In this case, before we glue $A N$ and $L B$ along $L M N$, we must choose a homotopy between our two structures on $L M N$. Thus we actually glue a copy of $L M N \times I$ in between $A N$ and $L B$. $A$ priori, the choice of homotopy could affect the value of the Massey product. In all interesting cases (in particular for symplectic cobordism), $B$ will have enough "higher homotopies" that the choice will not matter.

4. The Pontrjagin-Thom map. In this section we investigate how the Massey product behaves in the Pontrjagin-Thom theorem. We put a secondary structure on $\pi_{*}(M B)$ and show that the Pontrjagin-Thom map $\Phi: \Omega^{B} \rightarrow \pi_{*}(M B)$ is a map of secondary structures. This result holds even in categories of manifolds where $\Phi$ is not an isomorphism (it does not use transversality), but if $\Phi$ is an isomorphism, it changes the computation of secondary products into a homotopy problem. 
Let $M B=\{M B(n)\}$ denote the Thom spectrum of $B$. (See for example [16, Chapter II].) $\mu_{B}: B(n) \times B\left(n^{\prime}\right) \rightarrow B\left(n+n^{\prime}\right)$ induces a pairing $\mu_{B}: M B \times M B \rightarrow M B$. Let $f, g, h$ represent elements of $\pi_{l}(M B), \pi_{m}(M B), \pi_{n}(M B)$ respectively. Thus, e.g., $f: S^{l} \rightarrow M B$ is a sequence of maps $f_{r}: S^{l+r} \rightarrow M B(r)$ commuting with the various connecting maps. Suppose

$$
f \wedge g: S^{l+m}=S^{l} \wedge S^{m} \rightarrow M B, \quad g \wedge h: S^{m+n} \rightarrow S^{m} \wedge S^{n} \rightarrow M B
$$

are null-homotopic. Let $I=[0,1]$ and $C S^{k}=(I, 1) \wedge\left(S^{k}, *\right)=I \times S^{k} / 1 \times S^{k} \cup I \times *$ be the cone on $S^{k}$. There exist maps $F: C S^{l+m} \rightarrow M B, G: C S^{m+n} \rightarrow M B$ such that $F(0, x)=(f \wedge g) x, G(0, x)=(g \wedge h) x$. Then

$$
F \wedge h:(I, 1) \wedge\left(S^{l+m}, *\right) \wedge\left(S^{n}, *\right) \rightarrow(M B, *),
$$

and if $T:(I, 1) \wedge\left(S^{l}, *\right) \rightarrow\left(S^{l}, *\right) \wedge(I, 1)$ switches factors,

$$
(f \wedge G) T:(I, 1) \wedge\left(S^{l}, *\right) \wedge\left(S^{m+n}, *\right) \rightarrow(M B, *) .
$$

Since $(f \wedge g) \wedge h=f \wedge(g \wedge h)$,

$$
(F \wedge h)(0, x)=(f \wedge g \wedge h)(x)=(f \wedge G) T(0, x) \text { for } x \in S^{l+m+n} .
$$

We define $H: S^{l+m+n+1}=([-1,1], 1 \cup-1) \wedge\left(S^{l+m+n}, *\right) \rightarrow(M B, *)$ by

$$
\begin{aligned}
H(t, x) & =(F \wedge h)(t, x) & & \text { if } t \geqq 0, \\
& =(f \wedge G)(-t, x) & & \text { if } t \leqq 0 .
\end{aligned}
$$

4.1. Definition. If, in $\pi_{*}(M B),[f]=a,[g]=b,[h]=c$, let $\langle a, b, c\rangle=$ the set of all $[H]$.

We leave it to the reader to verify it is well defined and also to verify the following result.

4.2. Proposition. $\langle,$,$\rangle is a secondary product structure on \pi_{*}(M B)$ of degree +1 . If $B$ is commutative, so is the secondary structure.

We now look at $\Phi: \Omega^{B} \rightarrow \pi_{*}(M B)$.

4.3. THEOREM. $\Phi$ is a map of rings with secondary products.

Proof. Let $\left(L \iota_{L}, \xi_{L}\right),\left(M, \iota_{M}, \xi_{M}\right),\left(N, \iota_{N}, \xi_{N}\right)$ be three closed $B$-manifolds with $L M=\partial A, A=\left(A, \iota_{A}, \xi_{A}\right), M N=\partial C, C=\left(C, \iota_{C}, \xi_{C}\right)$. Then

$$
-\iota_{B}: C \rightarrow H^{-} \times E^{m+n+k_{2}+k_{3}-1} \text {. }
$$

The Thom construction (i.e., collapse of $E^{R}$ outside of a tubular neighborhood of the image of an embedding) gives maps

$$
f_{L}: S^{l+k_{1}} \rightarrow M B\left(k_{1}\right), \quad g_{M}: S^{m+k_{2}} \rightarrow M B\left(k_{2}\right), \quad g_{N}: S^{n+k_{3}} \rightarrow M B\left(k_{3}\right) .
$$

The tubular neighborhoods of $\iota_{A}(A), \iota_{C}(C)$ can be assumed to intersect

$$
\begin{aligned}
& E^{l+m+k_{1}+k_{2}}=0 \times E^{l+m+k_{1}+k_{2}} \subset H^{+} \times E^{l+m+k_{1}+k_{2},} \\
& E^{m+n+k_{2}+k_{3}}=0 \times E^{m+n+k_{2}+k_{3}} \subset H^{+} \times E^{m+n+k_{2}+k_{3}}
\end{aligned}
$$


orthogonally and so that their intersections with $E^{l+m+k_{1}+k_{2}}, E^{m+n+k_{2}+k_{3}}$ are the products of the tubular neighborhoods of $\iota_{L}(L), \iota_{M}(M), \iota_{N}(N)$. Then we also get

$$
f_{A}: C S^{l+m+k_{1}+k_{2}} \rightarrow M B\left(k_{1}+k_{2}\right), \quad f_{C}: C S^{m+n+k_{2}+k_{3}} \rightarrow M B\left(k_{2}+k_{3}\right) .
$$

Emulating the construction leading to 4.1, we get maps

$$
\begin{aligned}
f_{A} & \wedge f_{N}: C S^{l+m+n+k_{1}+k_{2}+k_{3}} \rightarrow M B\left(k_{1}+k_{2}+k_{3}\right), \\
\left(f_{L} \wedge f_{B}\right) T: C S^{l+m+n+k_{1}+k_{2}+k_{3}} & \rightarrow M B\left(k_{1}+k_{2}+k_{3}\right)
\end{aligned}
$$

agreeing on $S^{l+m+n+k_{1}+k_{2}+k_{3}}$. We glue them together to get a map

$$
H: S^{l+m+n+1+k_{1}+k_{2}+k_{3}} \rightarrow M B\left(k_{1}+k_{2}+k_{3}\right) \text {. }
$$

It is clear that as we vary $k_{1}, k_{2}, k_{3}$, this is precisely the construction of 4.1 . Hence

$$
\Phi\langle[L],[M],[N]\rangle \subset\langle\Phi[L], \Phi[M], \Phi[N]\rangle .
$$

5. The Adams spectral sequence. The main computational tool we develop to handle the homotopy problem of the last section is the Adams spectra sequence. We put secondary products in the Adams sequence and determine some of their properties.

We briefly recall the Adams spectral sequence [1]. For each prime $p$, there exists a spectral sequence $E_{r}^{s, t}(s \geqq 0, t \geqq s)$, of total grading $t-s$, with differentials $d_{r}: E_{r}^{s, t} \rightarrow E_{r}^{s+r, t+r-1}$ such that

$$
E_{2}^{s, t} \approx \operatorname{Ext}_{A}^{s, t}\left(H^{*}\left(M B ; Z_{p}\right) ; Z_{p}\right) \quad(A=\bmod p \text { Steenrod algebra }),
$$

$E_{r}$ converges weakly to $\pi_{*}(M B) /($ elements of finite order prime to $p)$. Furthermore, the pairing $M B \wedge M B \rightarrow M B$ determines a coproduct over $A$

$$
H^{*}\left(M B ; Z_{p}\right) \rightarrow H^{*}\left(M B ; Z_{p}\right) \otimes H^{*}\left(M B ; Z_{p}\right)
$$

and hence a pairing

$$
\begin{aligned}
& \operatorname{Ext}_{A}\left(H^{*}\left(M B ; Z_{p}\right) ; Z_{p}\right) \otimes \operatorname{Ext}_{A}\left(H^{*}\left(M B ; Z_{p}\right) ; Z_{p}\right) \\
& \rightarrow \operatorname{Ext}_{A}\left(H^{*}\left(M B ; Z_{p}\right) ; Z_{p}\right) .
\end{aligned}
$$

It also induces a pairing $E_{r}^{s, t} \otimes E_{r}^{s^{\prime}, t^{\prime}} \rightarrow E_{r}^{s+s^{\prime}, t+t^{\prime}}$ which is consistent (at $E_{\infty}$ ) with the induced pairing $\pi_{*}(M B) \otimes \pi_{*}(M B) \rightarrow \pi_{*}(M B)$ and which agrees with (5.2) at $E_{2}$. (There are several sign conventions. We suppose that if $a_{i}$ has bidegree $\left(s_{i}, t_{i}\right)$ for $i=1,2$, the isomorphism (5.1) changes the sign of $a_{1} a_{2}$ by $(-1)^{\left(t_{1}-s_{1}\right)\left(t_{2}-s_{2}\right)}$.) Also each $d_{r}$ is a derivation.

The copairing (5.2) induces a pairing

$$
\operatorname{Hom}_{A}\left(\mathscr{R}, Z_{p}\right) \otimes \operatorname{Hom}_{A}\left(\mathscr{R}, Z_{p}\right) \rightarrow \operatorname{Hom}_{A}\left(\mathscr{R}, Z_{p}\right)
$$

for a resolution $\mathscr{R}$ of $H^{*}\left(M B ; Z_{p}\right)$. This, of course, is what leads to (5.3). We also have from (5.4) a Massey product structure in

$$
\operatorname{Ext}_{A}\left(H^{*}\left(M B ; Z_{p}\right) ; Z_{p}\right) .
$$


5.6. THEOREM. Each $E_{r}$ has a secondary product structure of degree $(-r+1$, $-r+2)$ with the properties

1. At $E_{2}$, this structure is the same (via (5.1)) with the Massey structure on $\operatorname{Ext}_{A}\left(H^{*}\left(M B ; Z_{p}\right) ; Z_{p}\right)$ (as in 2.2.3). If $a_{i}(i=1,2,3)$ have bidegrees $\left(s_{i}, t_{i}\right)$ the sign of $\left\langle a_{1}, a_{2}, a_{3}\right\rangle$ is changed by $(-1)^{\left(t_{1}-s_{1}\right)\left(t_{2}-s_{2}\right)\left(t_{3}-s_{3}\right)}$.

For $r>2$, the structure on $E_{r}$ is the Massey structure on $E_{r}=H\left(E_{r-1}\right)$.

2. Let $a, b, c$ be elements in $E_{r}$ of total degree $\alpha, \beta, \gamma$, bidegrees $\left(\alpha^{\prime}, \alpha^{\prime \prime}\right),\left(\beta^{\prime}, \beta^{\prime \prime}\right)$, $\left(\gamma^{\prime}, \gamma^{\prime \prime}\right)$. Suppose $\langle a, b, c\rangle$ exists in $E_{r}$. Then

$$
d_{r}\langle a, b, c\rangle \subset-\left\langle\left(d_{r} a a\right),\left(\begin{array}{cc}
b & 0 \\
(-1)^{\alpha} d_{r} b & b
\end{array}\right),\left(\begin{array}{c}
d_{r} c \\
(-1)^{\alpha+\beta} c
\end{array}\right)\right\rangle .
$$

3. Let $a, b, c$ be as in part 2. If

(a) all elements in the groups

$$
\begin{array}{ll}
E_{\alpha^{\prime}+\beta^{\prime}-n+1}^{n, \alpha+\beta+n+1}, & 0 \leqq n \leqq \alpha^{\prime}+\beta^{\prime}-r, \\
E_{\beta^{\prime}+\gamma^{\prime}-n+1}^{n, \beta+\gamma+n+1}, & 0 \leqq n \leqq \beta^{\prime}+\gamma^{\prime}-r,
\end{array}
$$

are permanent cocycles and

(b) $a, b, c$ are permanent cocycles which in $E_{\infty}$ represent elements $f, g, h$ $\in \pi_{*}(M B)$ such that $\langle f, g, h\rangle$ is defined, and

(c) $\langle a, b, c\rangle$ is defined in $E_{r}$,

then there exist $F \in\langle f, g, h\rangle, \rho \in\langle a, b, c\rangle$ such that $\rho$ is a permanent cocycle and at $E_{\infty}$ represents $F$.

5.10. Note. For reference, we define matrix Massey products. Let $(C, \partial)$ be a graded (resp. bigraded) associative differential ring, with $\partial$ of degree $k= \pm 1$ (resp. bidegree $\left(k^{\prime}, k^{\prime \prime}\right)$ with $\left.k^{\prime}+k^{\prime \prime}= \pm 1\right)$. Let $A=\left(a_{i}\right), B=\left(b_{i j}\right), \Gamma=\left(c_{j}\right)$ be matrices over $H(C)$ of sizes $(1 \times m),(m \times n),(n \times 1)$ resp. Let grading $a_{i}=\left(\alpha_{i}\right)\left(\right.$ resp. $\left.\left(\alpha_{i}^{\prime} ; \alpha_{i}^{\prime \prime}\right)\right)$, grading $b_{i j}=\beta_{i j}\left(\operatorname{resp} .\left(\beta_{i j}^{\prime}, \beta_{i j}^{\prime \prime}\right)\right)$, grading $c_{j}=\gamma_{j}\left(\operatorname{resp} .\left(\gamma_{j}^{\prime}, \gamma_{j}^{\prime \prime}\right)\right)$. These matrices are to satisfy:

1. (a) For each $j$, there exists an integer $\mu_{j}$ (resp. pair of integers $\left.\left(\mu_{j}^{\prime}, \mu_{j}^{\prime \prime}\right)\right)$ such that $\alpha_{i}+\beta_{i j}=\mu_{j}$ for all $i$ (resp. $\left.\left(\mu_{j}^{\prime}, \mu_{j}^{\prime \prime}\right)=\left(\alpha_{i}^{\prime}, \alpha_{i}^{\prime \prime}\right)+\left(\beta_{i j}^{\prime}, \beta_{i j}^{\prime \prime}\right)\right)$.

(b) For each $i$, there exists an integer $\nu_{i}$ (resp. pair of integers $\left.\left(\nu_{i}^{\prime}, \nu_{i}^{\prime \prime}\right)\right)$ such that $\beta_{i j}+\gamma_{j}=\nu_{i}$ for all $j\left(\right.$ resp. $\left.\left(\nu_{i}^{\prime}, \nu_{i}^{\prime \prime}\right)=\left(\beta_{i j}^{\prime}, \beta_{i j}^{\prime \prime}\right)+\left(\gamma_{j}^{\prime}, \gamma_{j}^{\prime \prime}\right)\right)$. (Let $\lambda=\mu_{j}+\gamma_{j}=\alpha_{i}+\nu_{i}$ for any (=all) $i, j\left(\right.$ resp. $\left.\left(\lambda^{\prime}, \lambda^{\prime \prime}\right)=\left(\mu_{j}^{\prime}, \mu_{j}^{\prime \prime}\right)+\left(\gamma_{j}^{\prime}, \gamma_{j}^{\prime \prime}\right)=\left(\alpha_{i}^{\prime}, \alpha_{i}^{\prime \prime}\right)+\left(\nu_{i}^{\prime}, \nu_{j}^{\prime \prime}\right)\right)$.)

2. $A B=0, B \Gamma=0$.

Let $\bar{A}=\left(\bar{a}_{i}\right)$, etc., where $\bar{a}_{i}$ is a representative in $C$ of $a$. We have $\sum_{i} \bar{a}_{i} \bar{b}_{i j}=\partial \bar{x}_{j}$, $\sum_{j} \bar{b}_{i j} \bar{c}_{j}=(-1)^{\alpha_{i}} \partial \bar{y}_{i}$ for some $\bar{x}_{j}, \bar{y}_{i} \in C$. Let $\bar{X}=\left(\bar{x}_{j}\right), \bar{Y}=\left(\bar{y}_{i}\right)$. Let $\langle A|B| \Gamma\rangle$ be the classes of all elements $\bar{X} \bar{\Gamma}-\bar{A} \bar{Y}$ as $\bar{X}, \bar{Y}$ range over all possibilities. $\langle A|B| \Gamma\rangle$ $\subset H_{\lambda+k}(C)$ (resp. $H_{\lambda^{\prime}+k^{\prime}, \lambda^{\prime \prime}+k^{\prime \prime}}(C)$ ) and is a coset of the subgroup

$$
\begin{aligned}
\sum_{i} a_{i} H_{v_{i}+k}(C)+\sum_{j} H_{\mu_{j}+k}(C) c_{j} & \\
& \quad\left(\text { resp. } \sum_{i} a_{i} H_{v_{i}^{\prime}+k^{\prime}, v_{i}^{\prime}+k^{\prime \prime}}(C)+\sum_{j} H_{\mu_{j}^{\prime}+k, \mu_{j}+k^{\prime \prime}}(C) c_{j}\right) .
\end{aligned}
$$


Formula (5.7) holds in the following form: Let $\tilde{d}_{r}\left(b_{i j}\right)=\left((-1)^{\alpha_{i}} d_{r} b_{i j}\right), \tilde{d}_{r}\left(c_{j}\right)$ $=\left((-1)^{\mu}, d_{r} c_{j}\right)$. Then

$$
d_{r}\langle A, B, \Gamma\rangle \subset-\left\langle\left(d_{r} A A\right),\left(\begin{array}{cc}
B & 0 \\
\tilde{d}_{r} B & B
\end{array}\right),\left(\begin{array}{c}
\Gamma \\
\tilde{d}_{r} \Gamma
\end{array}\right)\right\rangle .
$$

The analogue of part 3 holds if parts (b), (c) are replaced by their obvious analogues and part (a) is replaced by $\left(a^{\prime}\right)$ : All elements in the groups

$$
\begin{array}{lll}
E_{\mu_{j}^{\prime}-n+1}^{n, \mu_{j}^{\prime}+\mu_{j}^{\prime}+n+1}, & \left(0 \leqq n \leqq \mu_{j}^{\prime}-r\right) & \text { for all } j, \\
E_{v_{i}^{\prime}-n+1}^{n, v_{i}^{\prime}+v_{i}^{\prime}+n+1}, & \left(0 \leqq n \leqq \nu_{i}^{\prime}-r\right) & \text { for all } i,
\end{array}
$$

are permanent cocycles.

5.11. Remarks. 1. The theorem is formally like analogous theorems of May [9] and Moss [10] although this situation is not formally contained in either of theirs.

2. Some conditions in part 3 are necessary for convergence to hold. The counterexample discussed in [10] may be considered to live in framed cobordism.

3. As the referee has remarked, it would be worthwhile to have more complete analogues of [9, Theorems 4.3, 4.5]. I have not been able to push through the details. Furthermore, our Theorem 5.6 suffices for the purposes of detecting Massey products.

4. We consider only the classical Adams spectral sequence, although parts of the theorem immediately go through for more general Adams sequences.

Proof of Theorem 5.6. For clarity, we work in the external situation. Suppose we have given six spectra $X_{1}, \ldots, X_{6}$ and pairings $\mu_{12}: X_{1} \wedge X_{2} \rightarrow X_{4}, \mu_{23}: X_{2} \wedge X_{3}$ $\rightarrow X_{5}, \mu_{15}: X_{1} \wedge X_{5} \rightarrow X_{6}, \mu_{43}: X_{4} \wedge X_{3} \rightarrow X_{6}$ such that

$$
\mu_{43}\left(\mu_{12} \wedge 1\right)=\mu_{15}\left(1 \wedge \mu_{43}\right): X_{1} \wedge X_{2} \wedge X_{3} \rightarrow X_{5} .
$$

The Adams sequence is constructed as follows: by using mapping cylinders if necessary, we may assume for each spectrum $X_{i}$, we have a sequence $\mathscr{R}$ of subspectra $X_{i}(p)$ and inclusions

$$
\rightarrow X_{i}(p) \rightarrow X_{i}(p-1) \rightarrow \cdots \rightarrow X_{i}(0)=X_{i} .
$$

Let $X_{i}(\infty)=\bigcap X_{i}(p)$. We use the notations $X_{i}(p, q)$ to denote the pair $\left(X_{i}(p), X_{i}(q)\right)$ and $\pi_{n}^{i}(p, q)=\pi_{n}\left(X_{i}(p, q)\right)$ if $q \geqq p$. We let $\kappa: \pi^{i}(p, q) \rightarrow \pi^{i}\left(p^{\prime}, q^{\prime}\right)$ be the map induced by the inclusion if $(p, q) \geqq\left(p^{\prime}, q^{\prime}\right)$. We have the exact triangles (with coefficients $Z_{p}$ for $p$ prime)

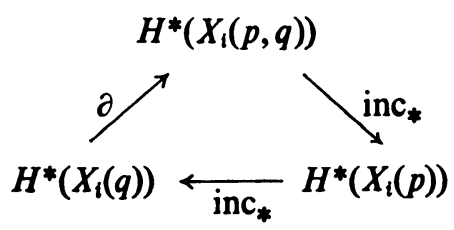


and the sequence

$$
\longrightarrow H^{*}\left(X_{i}(p, p+1)\right) \stackrel{\lambda_{p}}{\longrightarrow} H^{*}\left(X_{i}(p-1, p)\right) \longrightarrow \cdots \longrightarrow H^{*}\left(X_{i}(0,1)\right) \stackrel{\varepsilon}{\longrightarrow} H^{*}\left(X_{i}\right)
$$

with $\lambda_{p}=\partial$ inc $_{*}, \varepsilon=$ inc $_{*}$. It is to be a resolution of $H^{*}\left(X_{i}\right)$ over the $\bmod p$ Steenrod algebra $A$. We can assume that $\mu_{i j}\left(X_{i}(p) \wedge X_{j}(q)\right) \subset X_{k}(p+q)$ for whatever $i, j, k$ make sense. We have induced

Let

$$
\mu_{i j}\left(X_{i}(p, q) \wedge X_{j}\left(p^{\prime}, q^{\prime}\right)\right) \rightarrow X_{k}\left(p+p^{\prime}, \min \left(p+q^{\prime}, p^{\prime}+q\right)\right) .
$$

and

$$
\begin{aligned}
{ }_{i} Z_{r}^{p, q} & =\text { image }\left(\kappa: \pi_{q-p}^{i}(p, p+r) \rightarrow \pi_{q-p}^{i}(p, p+1)\right), \\
{ }_{i} B_{r}^{p, q} & =\text { image }\left(\partial: \pi_{q-p+1}^{i}(p-r+1, p) \rightarrow \pi_{q-p}^{i}(p, p+1)\right)
\end{aligned}
$$

$$
{ }_{i} E_{r}^{p, q}={ }_{i} Z_{r}^{p, q} \mid{ }_{i} B_{r}^{p, q} .
$$

$d_{r}$ is induced by $\partial: \pi(p, p+r) \rightarrow \pi(p+r, p+r+1) .\left({ }_{i} E_{r}, d_{r}\right)$ is the Adams spectral sequence.

To construct the secondary products, we emulate as closely as possible the construction of $\$ 4$. Let $a \in{ }_{1} E_{r}, b \in{ }_{2} E_{r}, c \in{ }_{3} E_{r}$ be such that $\langle a, b, c\rangle$ is defined in $E_{r}$; suppose they are represented respectively by maps

$$
\begin{gathered}
f:\left(I^{l}, I^{l}\right) \rightarrow X_{1}(p, p+r), \\
g:\left(I^{m}, I^{m}\right) \rightarrow X_{2}\left(p^{\prime}, p^{\prime}+r\right), \\
h:\left(I^{n}, I^{n}\right) \rightarrow X_{3}\left(p^{\prime \prime}, p^{\prime \prime}+r\right) .
\end{gathered}
$$

Consider the diagram

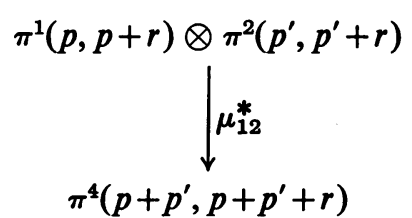

$$
\pi^{4}\left(p+p^{\prime}-r+1, p+p^{\prime}\right) \stackrel{\partial}{\longrightarrow} \pi^{4}\left(p+p^{\prime}, p+p^{\prime}+1\right) \stackrel{\kappa}{\longrightarrow} \pi^{4}\left(p+p^{\prime}-r+1, p+p^{\prime}+1\right) .
$$

The bottom row is exact. Since $\kappa \mu_{12}^{*}([f] \otimes[g]) \in \operatorname{im} \partial$,

$$
f \wedge g:\left(I^{l}, I^{l}\right) \wedge\left(I^{m}, I^{m}\right) \rightarrow X_{4}\left(p+p^{\prime}-r+1, p+p^{\prime}+1\right)
$$

is homotopic to *. (Here and elsewhere we make obvious abuses of notation.) There exists a map

$$
F:(I, 1) \wedge\left(I^{l}, I^{l}\right) \wedge\left(I^{m}, I^{m}\right) \rightarrow X_{4}\left(p+p^{\prime}-r+1, p+p^{\prime}+1\right)
$$

such that

$$
F \mid 0 \wedge\left(I^{l}, I^{l}\right) \wedge\left(I^{m}, I^{m}\right)=f \wedge g
$$


Similarly

$$
g \wedge h:\left(I^{m}, I^{m}\right) \wedge\left(I^{n}, I^{n}\right) \rightarrow X_{5}\left(p^{\prime}+p^{\prime \prime}-r+1, p^{\prime}+p^{\prime \prime}+1\right)
$$

is homotopic to $*$, so there exists

$$
G:(I, 1) \wedge\left(I^{m}, I^{m}\right) \wedge\left(I^{n}, I^{n}\right) \rightarrow X_{5}\left(p^{\prime}+p^{\prime \prime}-r+1, p^{\prime}+p^{\prime \prime}+1\right)
$$

such that

$$
G \mid 0 \wedge\left(I^{m}, I^{m}\right) \wedge\left(I^{n}, I^{n}\right)=g \wedge h .
$$

Let $T:(I, 1) \wedge\left(I^{l}, I^{l}\right) \rightarrow\left(I^{l}, I^{l}\right) \wedge(I, 1)$ switch factors. Let $P=p+p^{\prime}+p^{\prime \prime}$. Let

$$
\left(I^{l+m+n+1}, I^{l+m+n+1}\right)=([-1,1], 1 \cup-1) \wedge\left(I^{l+m+n}, I^{l+m+n}\right) .
$$

Define $H:\left(I^{l+m+n+1}, I^{l+m+n+1}\right) \rightarrow X_{6}(P-r+1, P+1)$ by

$$
\begin{aligned}
H(t, x) & =(F \wedge h)(t, x) & \text { if } t \geqq 0, \\
& =(f \wedge G) T(t, x) & \text { if } t \leqq 0,
\end{aligned}
$$

for $t \in[-1,1], x \in I^{l+m+n}=I^{l} \wedge I^{m} \wedge I^{n}$. The set of such $H$ determines a subset of $\pi^{6}(P-r+1, P+1)$ and hence a subset of ${ }_{6} Z^{P-r+1, P-r+2+l+m+n}$. The set of classes of such elements $H$ in $E_{r}$ we call $\langle a, b, c\rangle$.

We now reinterpret this construction in algebraic setting. Recall in (5.12), $\kappa \mu_{12}^{*}([f] \wedge[g]) \in$ image $\partial \subset \pi^{4}\left(p+p^{\prime}, p+p^{\prime}+1\right)$.

Let $\xi:\left(I^{l+m+1}, I^{l+m+1}\right) \rightarrow X_{4}\left(p+p^{\prime}, p+p^{\prime}+1\right)$ be such that $\partial[\xi]=\kappa \mu_{12}^{*}([f] \wedge[g])$. This means $f \wedge g:\left(I^{l+m}, I^{l+m}\right) \rightarrow X_{4}\left(p+p^{\prime}, p+p^{\prime}+1\right)$ is homotopic to a map $(f \wedge g)^{\prime}$ such that $(f \wedge g)^{\prime} \mid I^{l+m}: I^{l+m} \rightarrow *$, and hence $(f \wedge g)^{\prime}$ factors as in (5.16).

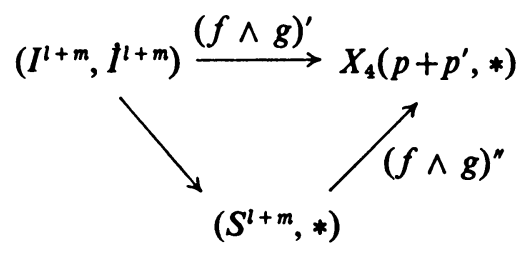

Furthermore, we may choose $\xi$ so that $\xi \mid I^{l+m+1}=(f \wedge g)^{\prime \prime}$ if we equate $I^{l+m+1}$ $=S^{l+m}$. If we patch $\xi$ and the homotopy between $f \wedge g,(f \wedge g)^{\prime}$ together by glueing along $\xi \mid I^{l+m+1}=(f \wedge g)^{\prime \prime}$, we find we have constructed an $F$ as in (5.13). Conversely any such $F$ is homotopic, with all stages of the homotopy satisfying (5.14), to such a construction.

Thus if $\xi$ represents $\bar{x}$ in ${ }_{4} E_{r-1}$, a similar $\eta$ represents $\bar{y}$ in ${ }_{5} E_{r-1}$, and $\bar{a}, \bar{b}, \bar{c}$ in ${ }_{6} E_{r}$ represent $a, b, c$, we immediately see that the classes $H$ in ${ }_{6} E_{r}$ are precisely those of the form class $\left(\bar{x} \bar{c}+(-1)^{l+1} \bar{a} \bar{y}\right)$. The sign $(-1)^{l}$ comes from the fact that $T$ changes orientation by $(-1)^{l}$; the other $(-1)$ comes from the change of $[0,1]$ to $[-1,0]$ in $(5.15)$.

Thus $\langle a, b, c\rangle$ is in fact a Massey product. It thus satisfies properties 2.1 automatically. 
The observant reader will note that we have put something over on him. In constructing our sequence of subspectra $X_{i}(p)$ we may have lost strict associativity and have only homotopy associativity in our products. Thus it may be necessary to modify (5.15) to accommodate the homotopy. This will still lead to some Massey product in the spectral sequence and, since there is only one consistent with the algebra, the complication makes no difference. We thus continue as if it were not there.

Part (1) of the theorem is proved for $r>2$. If we note that

$$
E_{1} \approx \operatorname{Hom}_{A}\left(H^{*}\left(\mathscr{R} ; Z_{p}\right) ; Z_{p}\right)
$$

as differential rings for a judicious choice of resolution $\mathscr{R}$, part (1) of the theorem is also proved for $r=2$.

We turn to the proof of part (2). In general, if $a \in E_{r}$ is represented by $f:\left(I^{l}, I^{l}\right) \rightarrow X(p, p+r), d_{r} a$ is represented by

$$
\partial f=f \mid I^{l}:\left(I^{l}, *\right) \rightarrow X(p+r, *) \rightarrow X(p+r, p+r+1) .
$$

Note that $\partial\left(I^{l} \times I^{m}\right)=\left(I^{l} \times I^{m}\right) \cup\left(I^{l} \times I^{m}\right)$ and that

$$
\partial(f \wedge g):\left(\left(I^{l} \times I^{m}\right) \cup\left(I^{l} \times I^{m}\right), *\right) \rightarrow X\left(p+p^{\prime}+r, p+p^{\prime}+r+1\right)
$$

breaks up so that $\partial(f \wedge g) \mid I^{l} \times I^{m}$ represents $d_{r} a \cdot b$ and $\partial(f \wedge g) \mid I^{l} \times I^{m}$ represents $(-1)^{\alpha} a \cdot d_{r} b$ with the induced orientations. We want to consider $\partial H:\left(I^{l+m+n+1}, *\right)$ $\rightarrow X_{6}(P+1, P+r+1)$. Using (5.15), it breaks up into

$$
\begin{aligned}
& \text { (5.17) } \partial(F \wedge h):(I, 1) \wedge \partial\left(\left(I^{l+m}, I^{l+m}\right) \wedge\left(I^{n}, I^{n}\right)\right) \rightarrow X_{6}(P+1, P+r+1), \\
& \text { (5.18) } \partial(f \wedge G) T:(I, 1) \wedge \partial\left(\left(I^{l}, I^{l}\right) \wedge\left(I^{m+n}, I^{m+n}\right)\right) \rightarrow X_{6}(P+1, P+r+1) .
\end{aligned}
$$

We break $\partial(F \wedge h)$ into

$$
\begin{gathered}
\partial(F \wedge h) \mid\left(I \times I^{l+m}\right) \times I^{n}, \\
\partial(F \wedge h) \mid I \times I^{l+m} \times I^{n} .
\end{gathered}
$$

(5.19) is a cone on a representative of $d_{r}(a b)$ times a representative of $c$. (5.20) is a cone on a representative of $a b$ times a representative of $d_{r} c$. (5.18) similarly breaks up. The union corresponds to sum. Checking the signs due to orientation reversals, we find that $\partial H$ is a representative for the matrix Massey product (5.7).

To prove part (3), consider the diagram (5.21):

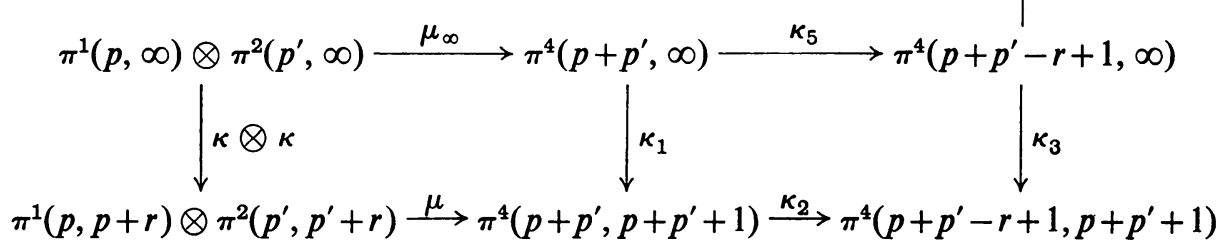


The various $\kappa$ are induced by inclusions. Suppose $f \in \pi_{1}(p, \infty), g \in \pi_{2}\left(p^{\prime}, \infty\right)$. Condition (3b) means that

$$
\kappa_{4} \kappa_{5} \mu_{\infty}(f \otimes g)=0 .
$$

Condition (3c) means that

$$
\kappa_{3} \kappa_{5} \mu_{\infty}(f \otimes g)=0 .
$$

If we knew that

$$
\kappa_{\infty} \mu_{\infty}(f \otimes g)=0,
$$

we could perform the cone construction (5.13) in $X_{4}\left(p+p^{\prime}-r+1, \infty\right)$, and, with a similar analysis for $g \otimes h$, prove the result. To prove (5.24), we use a lemma of Moss [10, Proposition 6.3].

5.25. Lemma. Suppose in ${ }_{i} E,(5.8),(5.22),(5.23)$ hold and the spectral sequence is weakly convergent. Then (5.24) is true in ${ }_{i} E$.

This completes the proof of 5.6.

6. Massey products in $S U$ and $S p$. In this section, by way of illustration and application, we compute some of the secondary structure of $\Omega^{s v}$, the special unitary cobordism groups, and exhibit a certain multiplicatively indecomposable element in symplectic cobordism as a Massey product. $\Omega_{1}^{S U} \approx Z_{2}$ has as its nonzero element $\alpha$, represented by a framed $S^{1} . \Omega_{2}^{S U} \approx Z_{2}$ on $\alpha^{2} ; \Omega_{3}^{S U}=0$ so $\alpha^{3}=0$. The interesting products are those of the form $\left\langle\alpha, \alpha^{2}, x\right\rangle$ or $\langle 2, \alpha, x\rangle$. Before we proceed, we recall some of the structure of $\Omega^{S U}$. References are [3], [5], [16]. Let $y_{2} \in \Omega_{2}^{U}$ be a two dimensional generator of $\Omega^{U},\left(y_{2}\right)$ the ideal generated by it.

6.1. THEOREM. 1. The natural map

$$
\Omega^{S U} \otimes Z\left[\frac{1}{2}\right] \rightarrow \Omega^{U} /\left(y_{2}\right) \otimes Z\left[\frac{1}{2}\right]
$$

is an isomorphism.

2. Torsion $\Omega^{S U}=0$ if $n \not 1$ or $2 \bmod 8$. Torsion $\Omega_{8 k+1}^{S U}$, torsion $\Omega_{8 k+2}^{S U}$ are vector torsion spaces over $Z_{2}$ of dimension the number of partitions of $k$.

3. There exist elements $m_{8 k} \in \Omega_{8 k}^{S U}$ such that a basis for torsion $\Omega^{S U}$ is $\{\alpha \cdot$ monomials in $m_{8 k}, \alpha^{2} \cdot$ monomials in $\left.m_{8 k}\right\}$.

4. There exists a 4-dimensional generator $x_{4}$ such that $x_{4}^{2}=4 m_{8}$.

We collect the results into an omnibus theorem. They are representative of the type of results one can obtain using Massey products. Let $x_{6}$ generate $\Omega_{6}^{S U} \approx Z$.

6.2. THEOREM. 1. $\langle 2, \alpha, 2\rangle=\alpha^{2} \bmod 0$.

2. $\left\langle\alpha, \alpha^{2}, 2\right\rangle=x_{4} \bmod 2 \Omega_{4}^{S U}$.

3. If $2 x=0, \alpha x \in\langle 2, x, 2\rangle$. (A similar result is true in framed cobordism. See [17, Corollary 3.7].)

4. If $\alpha x=0$, for any $y \in\langle 2, \alpha, x\rangle, \alpha y=0$ and $\left\langle\alpha^{2}, \alpha, x\right\rangle \cap\langle 2 \alpha y\rangle \neq \varnothing$. 
5. If $\alpha x=0, x_{4} x$ is divisible by 2. In fact $\frac{1}{2} x_{4} x \subset\left\langle\alpha^{2}, \alpha, x\right\rangle$. (Note that $\frac{1}{2} x_{4} x$ is itself a coset of the torsion subgroup.)

6. indet $\left\langle 2, \alpha, x_{6}\right\rangle=2 \Omega_{8}^{S U}$. There exists $x_{8} \in\left\langle 2, \alpha, x_{6}\right\rangle$ such that $x_{8}, m_{8}$ generate $\Omega_{8}^{S U} \approx Z \oplus Z$.

7. indet $\left\langle\alpha^{2}, \alpha, x_{6}\right\rangle=$ torsion $\Omega_{10}^{S U}+x_{6} \cdot \Omega_{4}^{S U}$. All elements of $\left\langle\alpha^{2}, \alpha, x_{6}\right\rangle$ are indecomposable.

8. For any $x_{8}$ from 6.2.6, $\alpha x_{8}=0, \frac{1}{2} x_{4} x_{6} \cap\left\langle 2, \alpha, x_{8}\right\rangle \neq \varnothing$.

6.3. Remarks. 1. These results give a large number of elements, including some indecomposable ones, as Massey products.

2. 6.2.5 has several interesting corollaries. It gives, for instance,

$$
\left\langle\alpha, \alpha^{2}, x_{4}\right\rangle=\frac{1}{2} x_{4}^{2}=2 m_{8} \bmod x_{4} \cdot \Omega_{8}^{S U} .
$$

Also, it may be of some academic interest to note the following. In the mod 2 Adams spectral sequence for $\Omega^{S U}$, all group extensions at $E_{\infty}$ are determined by the multiplicative structure except for one collection of extensions, all similar. 6.2.5 determines those extensions. We discuss this further below.

3. N. Ray and R. M. Switzer, in [13, Lemma 4.2], have a result quite similar to 6.2.5. In fact, it can be proved exactly as in 6.2.5. A propos their use of their 4.2, we refer to 6.3.2.

Now we consider symplectic cobordism. $\Omega_{1}^{S p} \approx Z_{2}$ on generator $\sigma_{1}$. Also $\Omega_{5}^{S p} \approx Z_{2}$ on generator $\sigma_{2}$. Since $\Omega_{11}^{S p} \approx \Omega_{15}^{S p} \approx 0$, the product $\left\langle\sigma_{1}, \sigma_{2}^{2}, \sigma_{2}\right\rangle$ is defined in $\Omega_{17}^{S p}$. The group $\Omega_{17}^{S p} \approx Z_{2} \oplus Z_{2} \oplus Z_{2}$ and $\sigma_{1} \cdot \Omega_{16}^{S p}+\sigma_{2} \cdot \Omega_{12}^{S p} \approx Z_{2} \oplus Z_{2}$. Any element not in this last subgroup (which is indet $\left\langle\sigma_{1}, \sigma_{2}^{2}, \sigma_{2}\right\rangle$ ) is multiplicatively indecomposable. From the analysis in [14], specifically Proposition 3.3(iii), we get the next result.

6.4. THEOREM. $\left\langle\sigma_{1}, \sigma_{2}^{2}, \sigma_{2}\right\rangle=\left\{\lambda \in \Omega_{17}^{S p} \mid \lambda\right.$ is multiplicatively indecomposable $\}$.

The author knows of no more explicit representatives for these $\lambda$.

We now turn to the proof of 6.2.

6.2.3 follows immediately from $2.1 .3,2.1 .4,6.1 .3,6.2 .1$.

6.2.4 follows from 2.1.6 as follows. Note that $\langle\alpha, 2, \alpha\rangle \subset \Omega_{3}^{S U}=0$. Thus for any $y$,

$$
\alpha y=\alpha\langle 2, \alpha, x\rangle=\langle\alpha, 2, \alpha\rangle x=0 \bmod 0 .
$$

Changing $y$ changes $\langle 2, \alpha, y\rangle$ by $\alpha^{2}$.something. This follows from 2.1.2, 6.2.1. Now we apply 2.1.6 and get

$$
0 \in\left\langle\alpha^{2}, \alpha, x\right\rangle+\langle 2,0, x\rangle+\langle 2, \alpha, y\rangle
$$

for any $y$. The middle term is absorbed in the indeterminacies of the others and we get 6.2.4.

6.2.5 is similar. We have, from 6.2.2,

$$
\begin{aligned}
x_{4} x & =\left\langle 2, \alpha^{2}, \alpha\right\rangle x \bmod 2 x \cdot \Omega_{4}^{S U}, \\
& =2\left\langle\alpha^{2}, \alpha, x\right\rangle \bmod 2 x \cdot \Omega_{4}^{S U} .
\end{aligned}
$$


Every element in the set on the right-hand side of 6.4 is divisible by 2 . Hence $x_{4} x$ is divisible by 2 . Furthermore, $x \cdot \Omega_{4}^{S U} \subset$ indet $\left\langle\alpha^{2}, \alpha, x\right\rangle$. Therefore $\frac{1}{2} x_{4} x$ $c\left\langle\alpha^{2}, \alpha, x\right\rangle$.

6.2.8 follows immediately from $6.2 .4,6.2 .5,6.2 .6,6.2 .7$.

Parts 1, 2, 6, 7 follow from computations in the mod 2 Adams spectral sequence. We recall a portion of its structure. References are [3], [7].

6.6. LEMMA. For $t-s \leqq 11, E_{2}^{s, t}$ has ring generators $1 \in E_{2}^{0,0}, q_{0} \in E_{2}^{1,1}, h_{1} \in E_{2}^{1,2}$, $\tau \in E_{2}^{3,7}, \omega_{1} \in E_{2}^{4,12}, q_{2} \in E_{2}^{1,7}, z_{1} \in E_{2}^{0,8}, y \in E_{2}^{0,10}$ with relations $q_{0} h_{1}=0, h_{1}^{3}=0$, $h_{1} \tau=0, h_{2} y=0, \tau^{2}=q_{0}^{2} \omega_{1}$. The only nonzero differential is $d_{2}$. It is determined by

$$
d_{2} z_{1}=h_{1} q_{2} .
$$

We have the following correspondence between $\Omega^{S U}$ and representatives in $E_{\infty}$ :

\begin{tabular}{c||c|c|c|c|c|c}
$\Omega^{S U}$ & 2 & $\alpha$ & $x_{4}$ & $x_{6}$ & $m_{8}$ & $x_{8}$ \\
\hline$E_{\infty}$ & $q_{0}$ & $h_{1}$ & $\tau$ & $q_{2}$ & $\omega_{1}$ & $q_{0} z_{1}$
\end{tabular}

The results on Massey products we need are

6.8. LeMMA. 1. In $E_{2},\left\langle q_{0}, h_{1}, q_{0}\right\rangle=h_{1}^{2} \bmod 0$.

2. In $E_{2},\left\langle q_{0}, h_{1}, h_{1}^{2}\right\rangle=\tau \bmod 0$.

3. In $E_{3}=H\left(E_{2}\right),\left\langle q_{0}, h_{1}, q_{2}\right\rangle=q_{0} z_{1} \bmod 0$.

4. In $E_{3}=H\left(E_{2}\right),\left\langle h_{1}^{2}, h_{1}, q_{2}\right\rangle=z_{1}^{\prime} \bmod 0$, where $z_{1}^{\prime}$ is the residue of $h_{1}^{2} z_{1}$.

Along with 5.1.3, Lemmas 6.6, 6.8 immediately prove the rest of 6.2.

6.9. REMARK. To continue 6.3.2, we note that if $\lambda \in \Omega_{10}^{S U}$ is represented by $z_{1}^{\prime}$ in $E_{\infty}$, the multiplicative structure cannot determine whether $2 \lambda=0,2 \lambda=x_{4} x_{6}$ (which is represented by $\tau q_{2}$ ) or $2 \lambda=\alpha^{2} m_{8}$ (which is represented by $h_{1}^{2} \omega_{1}$ ) 6.2 .5 and 6.8.4 immediately determine this extension; $2 \lambda=x_{4} x_{6}$. Furthermore, all extensions which the primary multiplicative structure does not determine are of the same type. See [3].

We turn to the proof of 6.8. Parts 3,4 follow from 6.7. We leave them to the reader. To establish parts 1, 2, we must determine Massey products in $\operatorname{Ext}\left(H^{*}\left(M S U ; Z_{2}\right) ; Z_{2}\right)$. Through resolution degree 7 ,

$$
\operatorname{Ext}\left(H^{*}\left(M S U ; Z_{2}\right) ; Z_{2}\right) \approx H^{*}\left(C^{\#} ; Z_{2}\right) \text {. }
$$

Here $C^{\#}$ (following Liulevicius [7]) is the sub-Hopf-algebra of the mod 2 Steenrod algebra generated by $1, S q^{2}, Q_{j}(j=0,1,2, \ldots)\left(Q_{j}\right.$ are in Milnor's notation). This isomorphism respects the primary and secondary ring structures. The dual Hopfalgebra of $C^{\#}$ is $Z_{2}\left[\tau_{j}\right] / I, j=0,1,2, \ldots$, where $I$ is the ideal generated by $\tau_{0}^{4}, \tau_{i}^{2}$ $(i>0) . \tau_{j}$ is dual to $Q_{j}$, and $\tau_{0}^{2}$ is dual to $S q^{2}$. The diagonal is given by

$$
\begin{aligned}
& \Delta \tau_{j}=1 \otimes \tau_{j}+\tau_{j} \otimes 1, \quad j \neq 1, \\
& \Delta \tau_{1}=1 \otimes \tau_{1}+\tau_{0}^{2} \otimes \tau_{0}+\tau_{1} \otimes 1 .
\end{aligned}
$$

$H^{*}\left(C^{\#} ; Z_{2}\right)$ is the homology of the reduced cobar construction. (See $[2, \S 2.2]$.) 
We have the correspondence $q_{0}=$ class $\left[\tau_{0}\right], q_{2}=$ class $\left[\tau_{2}\right], h_{1}=$ class $\left[\tau_{0}^{2}\right]$. Hence $h_{1}^{2}=$ class $\left[\tau_{0}^{2} \mid \tau_{0}^{2}\right]$.

To establish 6.8.1, note that $\left[\tau_{0}^{2} \mid \tau_{0}\right]=\delta\left[\tau_{1}\right],\left[\tau_{0} \mid \tau_{0}^{2}\right]=\delta\left(\left[\tau_{1}\right]+\left[\tau_{0}^{3}\right]\right)$. Hence

$$
\left\langle q_{0}, h_{1}, q_{0}\right\rangle=\operatorname{class}\left(\left[\tau_{0} \mid \tau_{1}\right]+\left[\tau_{1} \mid \tau_{0}\right]+\left[\tau_{0}^{3} \mid \tau_{0}\right]\right) .
$$

However

$$
\delta\left[\tau_{1} \tau_{0}\right]=\left[\tau_{1} \mid \tau_{0}\right]+\left[\tau_{0} \mid \tau_{1}\right]+\left[\tau_{0}^{3} \mid \tau_{0}\right]+\left[\tau_{0}^{2} \mid \tau_{0}^{2}\right] .
$$

This is the formula which establishes 6.8.1. (It can be also proved by noting $h_{1}=q_{0} \cup_{1} q_{0}$. See $[2, \S 2.2]$.)

To establish 6.8.2, we first note that $\tau$ is the only nonzero element of $H^{3,7}\left(C^{\#}\right)$. We need only show that $\left\langle h_{1}^{2}, h_{1}, q_{0}\right\rangle$ is nonzero. We have that

$$
\left[\tau_{0}^{2}\left|\tau_{0}^{2}\right| \tau_{0}^{2}\right]=\delta\left(\left[\tau_{1} \mid \tau_{1}\right]+\left[\tau_{0}^{2} \mid \tau_{1} \tau_{0}\right]+\left[\tau_{1} \tau_{0}^{2} \mid \tau_{0}\right]\right) .
$$

Thus

(6.11) $\left\langle h_{1}^{2}, h_{1}, q_{0}\right\rangle=\operatorname{class}\left(\left[\tau_{1}\left|\tau_{1}\right| \tau_{0}\right]+\left[\tau_{0}^{2}\left|\tau_{1} \tau_{0}\right| \tau_{0}\right]+\left[\tau_{1} \tau_{0}^{2}\left|\tau_{0}\right| \tau_{0}\right]+\left[\tau_{0}^{2}\left|\tau_{0}^{2}\right| \tau_{1}\right]\right)$.

Now let $\psi_{1}, \ldots, \psi_{k}$ be monomials in the $\tau_{j}$. Define a character, char $\left[\psi_{1}|\cdots| \psi_{k}\right]$ $\in Z_{2}$, by

$$
\begin{aligned}
\operatorname{char}[] & =1 & & ([] \text { is the empty cochain }) ; \\
\operatorname{char}\left[\psi_{1}|\cdots| \psi_{k}\right] & =1 & & \text { if } \psi_{i}=\tau_{j_{i}} \text { for some } j_{i}, i=1, \ldots, k, \\
& =0 & & \text { otherwise. }
\end{aligned}
$$

We extend char additively to all cochains $\Psi$. In fact, it is a ring homomorphism.

6.12. Lemma. If $\Psi$ is a boundary, char $\Psi=0$. Hence char is well defined on $H^{*}\left(C^{\# ;} Z_{2}\right)$.

Proof. If $\Psi$ is not of the form

$$
\left[\tau_{j_{1}}|\cdots| \tau_{j_{i}} \tau_{j_{i}+1}|\cdots| \tau_{j_{k}}\right]
$$

$\delta \Psi$ contains no term $\left[\tau_{j_{1}}|\cdots| \tau_{j_{k}}\right]$ and char $\delta \Psi=0$. This follows from the explicit formulae (6.10). If $\Psi$ is of the form (6.13), it also follows from (6.10) that

$$
\begin{aligned}
\operatorname{char} \delta \Psi & =\operatorname{char}\left(\left[\tau_{j_{1}}|\cdots| \tau_{j_{i}}\left|\tau_{j_{i_{+1}}}\right| \cdots \mid \tau_{j_{k}}\right]+\left[\tau_{j_{1}}|\cdots| \tau_{j_{i_{1}}}\left|\tau_{j_{l}}\right| \cdots \mid \tau_{j_{k}}\right]\right) \\
& =0 .
\end{aligned}
$$

Thus the lemma is proved.

By (6.11), char $\left\langle h_{1}^{2}, h_{1}, q_{0}\right\rangle=1$; hence $\left\langle h_{1}^{2}, h_{1}, q_{0}\right\rangle \neq 0$. This completes the proof of 6.8.2 and 6.2.

6.14. Remark. One could also consider the Adams-Novikov spectral sequence using unitary cobordism [11, p. 861]. In this case, it is the action of $d_{3}$ that gives formula 6.2.2. 


\section{J. C. ALEXANDER}

\section{REFERENCES}

1. J. F. Adams, On the structure and applications of the Steenrod algebra, Comment. Math. Helv. 32 (1958), 180-214. MR 20 \#2711.

2. - On the non-existence of elements of Hopf invariant one, Ann. of Math. (2) 72 (1960), 20-104. MR 25 \#4530.

3. D. W. Anderson, E. H. Brown, Jr. and F. P. Peterson, SU-cobordism, KO-characteristic numbers, and the Kervaire invariant, Ann. of Math. (2) 83 (1966), 54-67. MR 32 \#6470.

4. P. E. Conner and E. E. Floyd, Differentiable periodic maps, Ergebnisse der Mathematik und ihrer Grenzgebiete, N.F., Band 33, Academic Press, New York; Springer-Verlag, Berlin, 1964. MR 31 \#750.

5. —- Torsion in SU-bordism, Mem. Amer. Math. Soc. No. 60 (1966). MR 32 \#6471.

6. D. Kraines, Massey higher products, Trans. Amer. Math. Soc. 124 (1966), 431-449. MR 34 \#2010.

7. A. Liulevicius, Notes on homotopy of Thom spectra, Amer. J. Math. 86 (1964), 1-16. MR 29 \#4060.

8. W. S. Massey, Some higher order cohomology operations, Sympos. Internacional de Topologia Algebraica, Universidad Nacional Autónoma de México and UNESCO, Mexico City, 1958, pp. 145-154. MR 20 \#4826.

9. J. P. May, Matric Massey products, J. Algebra 12 (1969), 533-568. MR 39 \#289.

10. R. M. F. Moss, Secondary compositions and the Adams spectral sequence, Math. Z. 115 (1970), 283-310. MR 42 \#1123.

11. S. P. Novikov, The methods of algebraic topology from the point of view of cobordism theory, Izv. Akad. Nauk SSSR Ser. Mat. 31 (1967), 855-951. (Russian) MR 36 \#4561.

12. G. J. Porter, Higher products, Trans. Amer. Math. Soc. 148 (1970), 315-345. MR 41 \#1053.

13. N. Ray and R. M. Switzer, On SU $\times$ SU bordism, Quart. J. Math. 21 (1970), 137-150.

14. D. M. Segal, On the symplectic cobordism ring, Comment. Math. Helv. 45 (1970), 159-169. MR 41 \#7699.

15. E. H. Spanier, Higher order operations, Trans. Amer. Math. Soc. 109 (1963), 509-539. MR 28 \#1622.

16. R. E. Stong, Notes on cobordism theory, Math. Notes, Princeton Univ. Press, Princeton, N. J.; Univ. of Tokyo Press, Tokyo, 1968. MR 40 \#2108.

17. H. Toda, Composition methods in homotopy groups of spheres, Ann. of Math. Studies, no. 49, Princeton Univ. Press, Princeton, N. J., 1962. MR 26 \#777.

18. H. Uehara and W. S. Massey, The Jacobi identity for Whitehead products, Algebraic Geometry and Topology, Princeton Univ. Press, Princeton, N. J., 1957, pp. 361-377. MR 19, 974.

Department of Mathematics, University of Maryland, College Park, Maryland 20742 